\title{
Emergence of Deletions during Treadmill Locomotion as a Function of Supraspinal and Sensory Inputs
}

\author{
Marina Martinez, ${ }^{1,2}$ Marius Tuznik, ${ }^{1}$ Hugo Delivet-Mongrain, ${ }^{1}$ and Serge Rossignol ${ }^{1,2}$ \\ ${ }^{1}$ Groupe de Recherche sur le Système Nerveux Central, Université de Montréal, Department of Physiology, and ${ }^{2}$ SensoriMotor Rehabilitation Research \\ Team, Canadian Institutes for Health Research, Montreal, Quebec, H3J 3J7, Canada
}

During locomotion, alternating activity of flexor and extensor muscles is largely regulated by a spinal neuronal network, the central pattern generator, the activity of which is modulated by peripheral and supraspinal inputs. In the absence of these modulatory inputs, for example during fictive locomotion after spinalization and curarization, spontaneous failures of motor activation (deletions) in a muscle can occur without perturbing the rhythmic cycle structure of the antagonists on the same side or the contralateral side. This suggests that the central pattern generator can maintain the locomotor period when motoneuron discharges fail in a given pool of motoneurons. Here we first examined whether such deletions could occur during real locomotion on a treadmill and determined their consequences on the overt locomotor pattern. We also evaluated the role of supraspinal and sensory inputs in modulating the occurrence of failures of rhythmic activity by comparing the same cats in the intact state, then after a partial spinal cord injury (SCI), and finally after a complete SCI at different treadmill speeds. We showed that deletions: (1) are absent in intact animals and occur only after SCI; (2) affect only flexor muscle activity; (3) neither perturb the timing of rhythmic activity of these muscles in subsequent cycles nor interfere with the timing of the ipsilateral and contralateral agonists and antagonists; (4) do not affect significantly the locomotor pattern kinematics; and (5) are sensitive to treadmill speed and lesion severity, suggesting a role for sensory and supraspinal inputs in stabilizing rhythmic output activity.

\section{Introduction}

In all species, the basic locomotor pattern is organized by a central pattern generator (CPG) that generates a bilateral alternating activity of the limbs with a proper activation order of flexor and extensor muscles at each joint level (Grillner, 1981). As shown during fictive locomotion in chemically paralyzed, decerebrate, and spinalized cats (Pearson and Rossignol, 1991), this locomotor pattern can be elicited in the absence of sensory inputs, pharmacological stimulation, or descending inputs. Despite progress, the functional structure of the CPG is far from clear in higher mammals. Observations of failures in CPG operation are useful when attempting to address this question. During fictive locomotion and scratch, spontaneous failures in the normally robust flexor and extensor activity have been reported. The most extensively described failures are the missing burst of activity of one or more expected rhythmic bursts of activity in motoneuron pools (so-called deletions) during the turtle scratch reflex (Stein and

Received March 14, 2013; revised June 5, 2013; accepted June 11, 2013.

Author contributions: M.M. and S.R. designed research; M.M. and H.D.-M. performed research; M.M. and M.T. analyzed data; M.M. wrote the paper.

This work was funded by a Canada Research Chair on the Spinal Cord, the SensoriMotor Rehabilitation Research Team (SMRRT) as part of the Regenerative Medicine and Nanomedicine Strategic Initiative of the Canadian Institutes for Health Research (CIHR), and as by an individual grant from the CIHR (to S.R.). M.M. was funded by postdoctoral fellowships from the SMRRT, Fonds Québécois de la Recherche sur la Nature et les Technologies (FQRNT), and CIHR. We thank P. Drapeau for his competent help in this work.

The authors declare no competing financial interests.

Correspondence should be addressed to Serge Rossignol, Faculté de médecine, Département de physiologie, C.P. 6128, succ., Université de Montréal, Centreville, Montréal H3C 3J7, Canada. E-mail: serge.rossignol@umontreal.ca. DOI:10.1523/JNEUROSCI.1126-13.2013

Copyright $\odot 2013$ the authors $\quad 0270-6474 / 13 / 3311599-07 \$ 15.00 / 0$
Daniels-McQueen, 2002) and cat fictive locomotion (LafreniereRoula and McCrea, 2005; Rybak et al., 2006). The observations that some missing bursts of activity in agonists during fictive locomotion could actually not affect the timing of rhythmic activity in the corresponding antagonists (Lafreniere-Roula and McCrea, 2005) and also did not disturb the rhythmic activity on the other side of the cord (Zhong et al., 2012) were intriguing. However, no study has investigated whether such failures in CPG operation could occur during treadmill locomotion when the sensory and supraspinal systems interact with the spinal CPG to regulate the locomotor output.

Here we analyzed spontaneous deletions occurring during treadmill locomotion in intact and spinal cord injured (SCI) cats. We first counted and characterized the deletions of electromyographic (EMG) activity of several hindlimb muscles during treadmill walking and evaluated their impact on the other muscles activity and on the locomotor pattern. Second, we evaluated the role of supraspinal inputs on the occurrence of deletions by performing sequentially a unilateral hemisection on the left side and then a complete SCI in the same animals to respectively isolate partially and then totally the CPG from the brain (Martinez et al., 2011, 2012b). Because cats previously submitted to a spinal hemisection are able to walk early after the complete SCI (Martinez et al., 2011, 2012a,b), we were able to investigate, in the same animal, whether decreasing and then abolishing supraspinal inputs could affect the number of deletions obtained in each hindlimb. Third, we evaluated the role of sensory inputs by comparing the percentage of deletions obtained at different treadmill speeds. Our results showed that nonresetting deletions of flexor 
activity occurred during treadmill locomotion, but only after SCI. These deletions had no impact on agonists and antagonists activity on the same side or on the contralateral side and did not affect significantly the locomotor output. The occurrence of deletions was also shown to be highly dependent on the level of supraspinal and sensory inputs.

\section{Materials and Methods}

Protocol. All procedures followed a protocol approved by the ethics committee at the Université de Montréal according to the Canadian Guide for the Care and Use of Experimental Animals. Adult female $(n=7)$ and male $(n=3)$ cats weighing from 2.5 to $4.8 \mathrm{~kg}$ were engaged in a dual spinal lesion paradigm. The procedures for EMG implantation, spinal lesions, EMG and kinematic recordings, and histology were similar to those described previously (Martinez et al., 2011, 2012a,b). All cats were chronically implanted with intramuscular electrodes to record EMG activity from the sartorius anterior (Srt), semitendinosus (St), vastus lateralis (VL), gastrocnemius lateralis (GL), gastrocnemius medialis (GM), and tibialis anterior (TA). During episodes of locomotion, cats were filmed from the left side and EMG activity was synchronized with kinematics. After control recording sessions $(n=2-3)$, all cats underwent a hemisection targeting the left side of the spinal cord at T10 and their locomotor performances were then assessed once a week for 3 weeks. A complete spinalization was achieved at T13 and hindlimb locomotion was evaluated as early as $24 \mathrm{~h}$ after spinalization and then once a week for 3 weeks.

Lesion size. In all cats, the hemisection was confined to the left side and always involved a significant portion of the left hemicord while sparing the most medial part of the left ventral funiculus, as described previously (Martinez et al., 2011; Martinez et al., 2012a). The second spinal lesion was complete in all cats.

Statistics. Data analyses were performed using Microsoft Excel and Sigmaplot 11.0 software to determine, in a sequence of locomotion, whether a deletion (i.e., missing burst) in a given muscle affected: (1) the rhythmic activity of this muscle (when the cycle period during the deletion was the same as in previous cycles, the deletion was considered as nonresetting); (2) the rhythmic activity and burst characteristics (duration and amplitude) of its agonists and antagonists on the same side and on the other side; (3) the locomotor pattern (kinematics; we used the $t$ statistics previously described by Lafreniere-Roula and McCrea, 2005 and Zhong et al., 2012). This method allowed us to compare a given parameter (e.g., burst duration) during the deletion period with the period preceding the deletion. A $p$-value $<0.05$ was considered statistically significant.

\section{Results}

\section{Deletions of flexor activity are observed during treadmill locomotion in SCI cats}

Records from 230 episodes of 17-20 consecutive step cycles of treadmill locomotion (30 in the intact state, 90 after partial SCI, and 110 after spinalization) obtained at the speed of $0.4-0.6 \mathrm{~m} / \mathrm{s}$ in 10 adult cats were examined. Each cat was sequentially tested at each speed $(0.4,0.5$, and $0.6 \mathrm{~m} / \mathrm{s})$ one time at the intact state $(3$ sequences per cat), 3 times after partial SCI ( 9 sequences per cat), and 3-4 times after spinalization (9-12 sequences per cat depending on their ability to walk immediately after spinalization). In the 30 episodes of locomotion evaluated at the intact state, disturbances in rhythmic activity were never reported. After the partial and complete SCI, over the 200 locomotor episodes examined, 69 episodes (i.e., 34.5\%) presented at least one example of a deletion in each cat that spanned one step cycle (referred to as single deletion) and only affected the St, Srt, and TA muscles. Although these muscles act most consistently as flexors during the early swing phase, St can also express more complex activity patterns (sometimes exhibiting two bursts per walking cycle). Specifically, single deletions of St were observed in 60/69 epi- sodes, whereas 6 and 3 episodes, respectively, contained single deletions of Srt and TA activity. In 9 episodes (i.e., 4.5\%), deletions spanned 2-3 step cycles (referred to as multicycle deletions) that only involved St activity. Given the high frequency of singlecycle deletions of the St muscle, only the consequences of these deletions will be described herein.

\section{Timing of rhythmic activity and burst components are maintained during deletions}

Impact of a St flexor deletion on St activity

We first investigated whether deletions were of nonresetting or resetting type. All single St deletions observed in the 60 episodes of locomotion were characterized as nonresetting. Figure $1 \mathrm{~A}$ gives an example of such a deletion in the right St occurring at the end of a bout of rhythmic activity in which the average interburst interval during the preceding cycles was $740 \mathrm{~ms}$ ( $\mathrm{SD}=71 \mathrm{~ms}, n=$ 17 cycles, see the horizontal gray stippled line representing the mean of 17 single-cycle periods). The interval between flexor bursts when the deletion occurred (gray rectangle) was 1522 ms - nearly twice the average value - and the deletion was thus characterized as a 2-cycle deletion. To determine whether the reemergence of St activity after the deletion occurred at the expected timing, the deletion duration was compared with the average of the 5 normal 2-cycle intervals immediately preceding the deletion (Fig. 1A, open rectangles) according to the statistical technique of Lafreniere-Roula and McCrea (2005) and Zhong et al. (2012) (see Materials and Methods). The deletion duration was not different from 2-cycle intervals in the control period preceding the deletion $(t=0.55, p=\mathrm{NS})$ such that the reemergence of the St activity after the deletion occurred at the expected time.

\section{Impact of a flexor deletion on agonists' activity}

Given the data showing that disturbances in rhythmic activity during fictive locomotion and scratch usually occur simultaneously in a set of synergist motoneurons (e.g., hindlimb flexors; Lafreniere-Roula and McCrea, 2005; Stein, 2008), we investigated whether a missing St burst during locomotion (a 2-cycle deletion) could affect the timing of rhythmic activity and burst components (duration and amplitude) in the other agonists (Srt and TA) on the same side. As shown in Figure $1 B$, the flexors Srt and TA exhibited continued rhythmic activity with no interruptions at the time of the missing St burst. The $t$ statistics comparing the cycle period of agonists (Srt and TA) 5 cycles before and during deletion showed that, during deletions of St activity, the other flexors maintained the same discharge frequency as before (Fig. $1 C ; p=\mathrm{NS}$ ). In addition, the burst duration (Fig. 1D) and amplitude (Fig. $1 E$ ) of agonists were not affected by the deletion.

\section{Impact of a flexor deletion on antagonists' activity}

As shown in previous studies, deletions in activity of one set of synergist motoneurons (e.g., hindlimb extensors) during fictive locomotion and scratch were usually accompanied by either sustained activity or continuing bursting in antagonist ipsilateral motoneurons (e.g., hindlimb flexors; Stein and DanielsMcQueen, 2002; Lafreniere-Roula and McCrea, 2005). We thus investigated whether a deletion of St flexor activity could affect the timing of rhythmic activity, burst duration, and amplitude in antagonists (GM, GL, VL) on the same side (Fig. $1 B^{\prime}$ ). During all of the St flexor deletions, the ipsilateral extensors (GM, GL, VL) maintained the same discharge frequency (Fig. $1 C^{\prime}$ ), burst duration (Fig. 1 $D^{\prime}$ ), and amplitude (Fig. $1 E^{\prime}$ ) as before. 


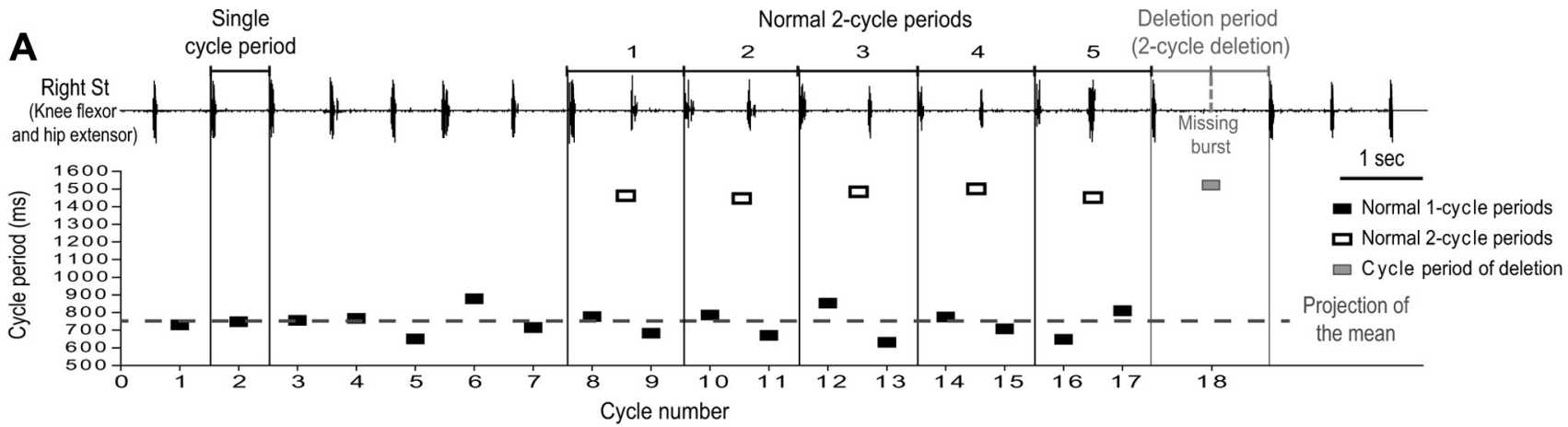

B

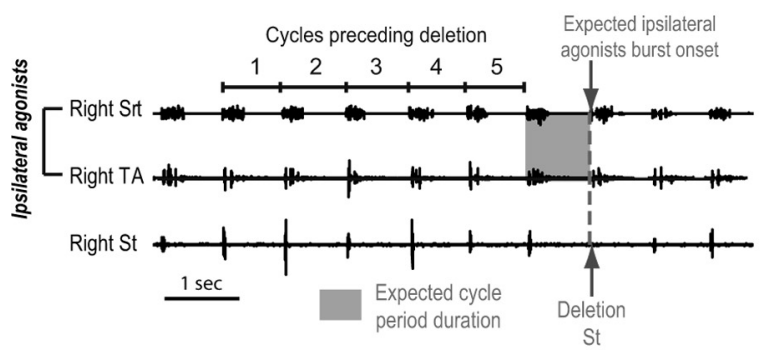

B'

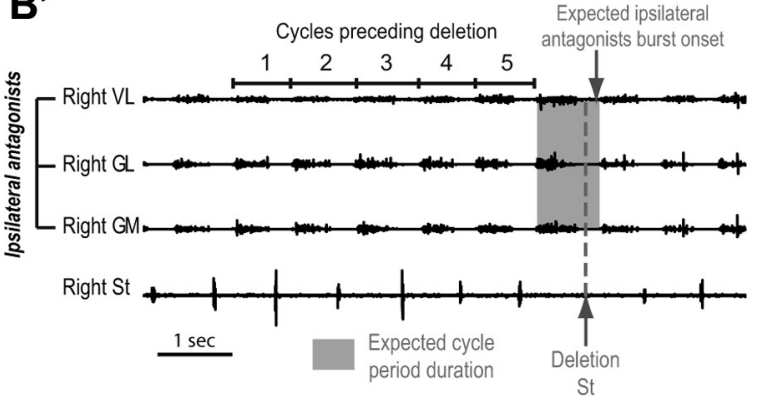

B"

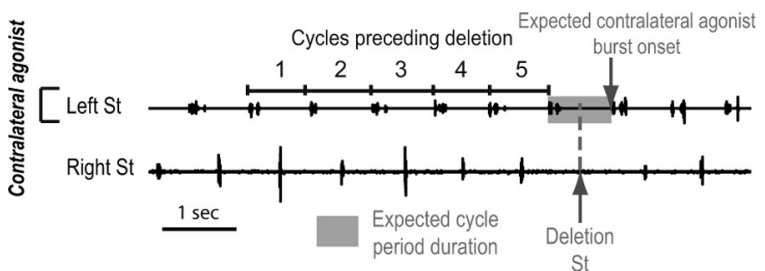

C
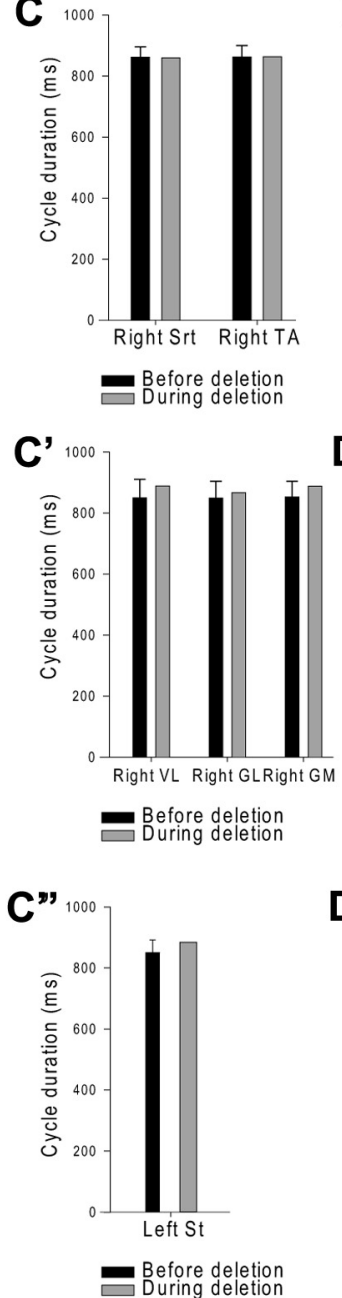

D ${ }^{350}$

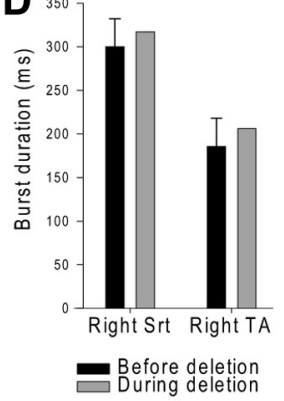

$E{ }^{0}$

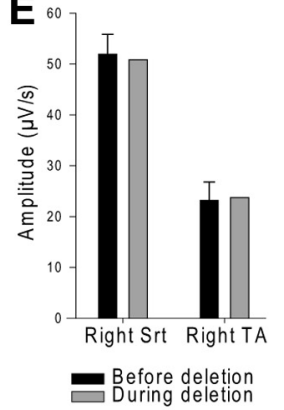

E'

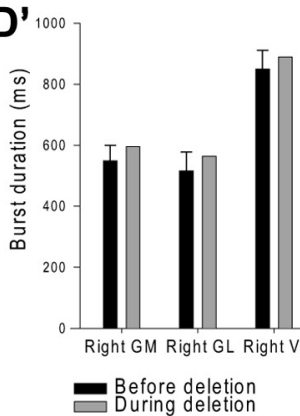

D" ${ }_{200}$

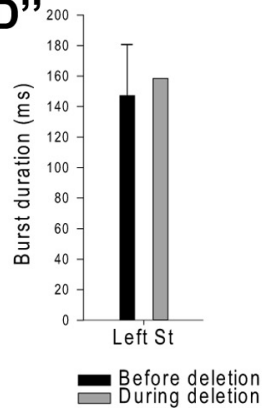

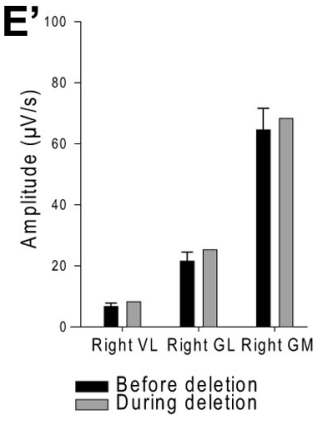

E'

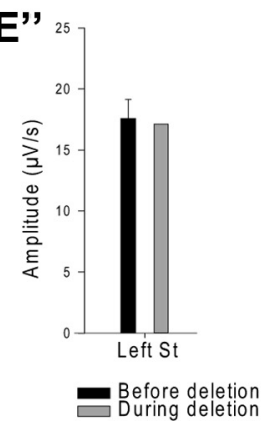

Figure 1. Maintenance of cycle timing and burst components during a deletion of knee flexor activity recorded during treadmill locomotion at $0.4 \mathrm{~m} / \mathrm{s} 1$ week after hemisection. $A, S t$ EMG traces in which a deletion occurs. Interval during the deletion (gray rectangle) was not different from normal 2-cycle intervals preceding the deletion (white rectangles; $t=0.55, \mathrm{p} \mathrm{n.s.).} \boldsymbol{B}-\boldsymbol{B}^{\prime \prime}, \mathbf{D}$ During a St deletion, activity was sustained in ipsilateral agonists (Srt, TA), antagonists (GM, GL, VL), and contralateral homologous agonists. In these muscles, the cycle duration $\left(\mathbf{C}-\boldsymbol{C}^{\prime \prime}\right)$, burst duration $\left(\boldsymbol{D}-\boldsymbol{D}^{\prime \prime}\right)$ and amplitude $\left(\boldsymbol{E}-\boldsymbol{E}^{\prime \prime}\right)$ during deletion (gray bars) was not different from the control period preceding the deletion (black bars). The bars above each set of traces show the expected timing of the bursts if the rhythm were unperturbed during the deletion.

Impact of a flexor deletion on contralateral muscle activity In a recent study using isolated mouse spinal cord preparations, nonresetting deletions occurring on one side of the cord were almost never accompanied by disturbances in rhythmic motor activity on the other side of the cord (Zhong et al., 2012). We thus investigated whether a deletion of St flexor activity could affect the timing of rhythmic activity within the contralateral St (Fig. $\left.1 B^{\prime \prime}\right)$. We found that a flexor deletion on one side did not affect the timing of rhythmic activity (Fig. $1 C^{\prime \prime}$ ) burst duration (Fig. $1 D^{\prime \prime}$ ), or amplitude (Fig. $1 E^{\prime \prime}$ ) of muscles on the other side.
To summarize, during treadmill locomotion in SCI cats, nonresetting deletions can occur in a flexor muscle without perturbing the rhythmic and bursting activity of this muscle or of the ipsilateral and contralateral agonists and antagonists.

Locomotor kinematics are maintained during deletions

We investigated whether St deletions induced disturbances in kinematics during treadmill locomotion in all episodes where the deletion occurred on the left side (i.e., 33 episodes). Normally, St discharges to initiate knee flexion. If a St deletion had 


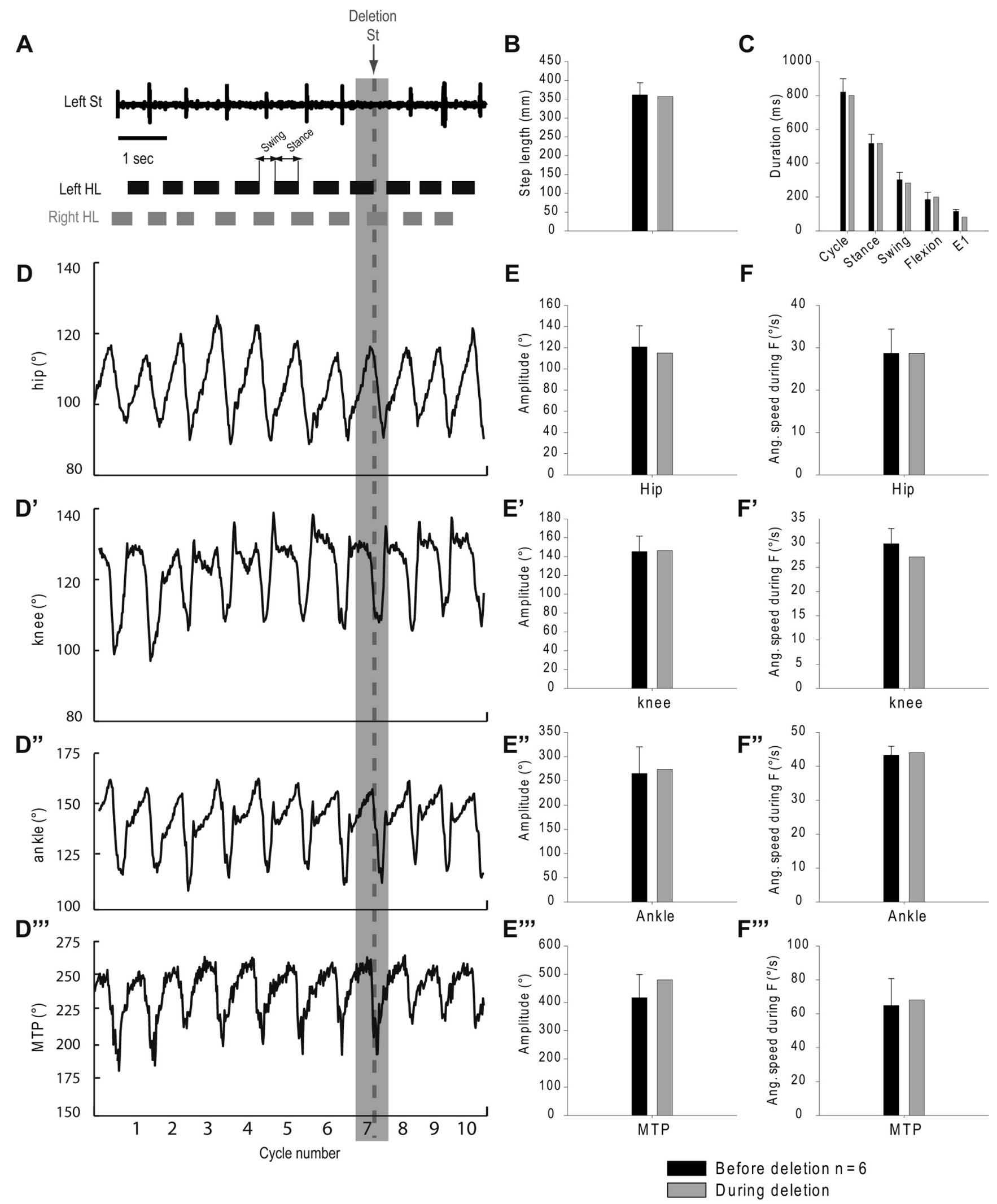

Figure 2. Maintenance of the left hindlimb locomotor pattern during a flexor deletion recorded during treadmill locomotion at $0.5 \mathrm{~m} / \mathrm{s} 1 \mathrm{~d}$ after spinalization in a cat previously submitted to a hemisection on the left side. $\boldsymbol{A}$, Left St EMG traces in which a deletion (gray dotted line) occurs. Duty cycles (horizontal bars) illustrate the support periods (stance) of each hindlimb. B, C, By comparison with the cycles preceding the deletion (black bars), the step length $(\boldsymbol{B})$, the duration of the cycle, of its subphases (stance and swing), and of the subphases of the swing (flexion and $E 1$ phases) ( $(\boldsymbol{C})$ were not affected by the deletion (gray bars). $\boldsymbol{D}-\boldsymbol{D}^{\prime \prime \prime}$, Angular excursion of the hip $(\boldsymbol{D})$, knee $\left(\boldsymbol{D}^{\prime}\right)$, ankle $\left(\boldsymbol{D}^{\prime \prime}\right)$, and MTP $\left(\boldsymbol{D}^{\prime \prime \prime}\right)$ before, during (gray rectangle), and after St deletion. $\boldsymbol{E}-\boldsymbol{F}^{\prime \prime \prime}$, By comparison with the cycles preceding the deletion (black bars), the amplitude of each joint $\left(\boldsymbol{E}-\boldsymbol{E}^{\prime \prime \prime}\right)$ and the angular speed of each joint during the flexion phase of the swing (gray bars; $\left.\boldsymbol{F}-\boldsymbol{F}^{\prime \prime \prime}\right)$ were not affected by the deletion. $\mathrm{HL}$, hindlimb; E1, first extension phase of the swing; Ang. speed during $\mathrm{F}$, angular speed during the flexion phase of the swing. 

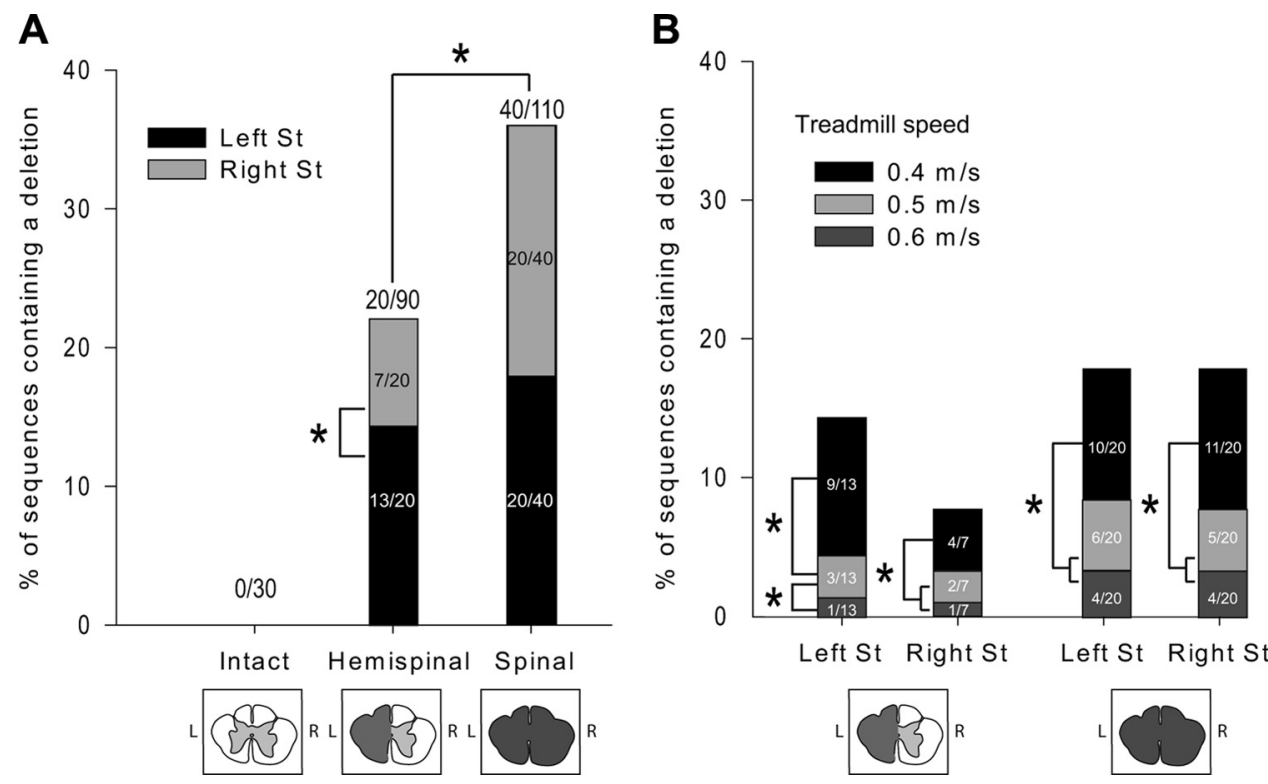

Figure 3. The occurrence of deletions is influenced by lesion size and treadmill speed. $A$, Comparison of the percentage and ratio of sequences containing a deletion in the left and right $S t$ in the intact state, after hemisection, and after a further complete spinalization. $\boldsymbol{B}$, Comparison of the percentage and ratio of sequences containing a deletion in the left and right $\mathrm{St}$ as a function of treadmill speed $(0.4-0.6 \mathrm{~m} / \mathrm{s})$ after hemisection and spinalization. Statistical differences between percentages are indicated by asterisks. Schematic drawings above the histogram represent the thoracic spinal cord at the intact, hemispinal, and spinal states. The damaged tissue is represented in gray. L, left; $R$, right.

an impact on locomotor pattern, one could expect that changes in kinematic parameters may occur during the deletion period and would especially affect the swing phase (i.e., flexion phase of the swing, knee amplitude, and knee angular speed) that in turn may have consequences on other kinematic parameters (i.e., step length, amplitude and angular speed of other joints, cycle and subphase duration). In all episodes analyzed, we showed that during deletions of St activity on the left side (Fig. 2A), the step cycle structure on the same side and on the other side (not illustrated) remained unchanged such that the step length (Fig. 2B), cycle, and subphase duration (Fig. $2 C$ ) were similar before and during the deletion. Moreover, the angular amplitude and speed of the hip (Fig. 2D-F), knee (Fig. $2 D^{\prime}-F^{\prime}$ ), ankle (Fig. $2 D^{\prime \prime}-F^{\prime \prime}$ ), and MTP (Fig. $2 D^{\prime \prime \prime}-$ $\left.F^{\prime \prime \prime}\right)$ were not affected ( $p=\mathrm{NS}$ ) and the forward and backward placement of the paw at contact and lift were also unchanged (data not shown; $p=\mathrm{NS}$ ). In summary, a deletion of St activity had no obvious kinematic consequences.

\section{Role of supraspinal and sensory inputs on the occurrence of deletions}

As demonstrated previously, supraspinal and peripheral sensory signals from the skin, muscles, and joints critically adapt the CPG to the changing circumstances of the environment (Rossignol et al., 2006). To study the potential role of supraspinal and sensory inputs in modulating the occurrence of deletions, we compared the ratio of deletions occurring on each side at different treadmill speeds $(0.4-0.6 \mathrm{~m} / \mathrm{s})$ by comparing the intact and partial spinal states and the partial and the complete spinal states.

Although we found no deletion in the intact state, $22 \%$ of the episodes examined after the partial SCI presented single deletions of St activity (20/90 episodes; Fig. 3A). Specifically, by comparing the ratio of deletions occurring on the left (lesion) and right (intact) sides, we showed that, after hemisection, the deletions were more frequently observed in left St
(13/20 episodes; Fig. $3 A$, black) compared with the right St (7/20; Fig. $3 A$, gray; $\chi^{2}$ test, $\left.p<0.01\right)$. The rhythmic pattern was thus more preserved on the side of the cord receiving direct (and probably modified) supraspinal inputs than on the lesion side that received fewer and more indirect inputs from the supraspinal structures. After complete spinalization, the number of deletions reached $36 \%$ of the episodes (40/110) and was statistically higher than the $22 \%$ observed after hemisection ( $\chi^{2}$ test, $p<0.05$ ), again suggesting a strong regulatory role of the suprapinal inputs. In contrast to the hemispinal period, during which the ratio of St deletions was different between sides, the ratio of deletions observed between the left and right sides after spinalization was similar in each side (20/40 for the left and right St; $\chi^{2}$ test, $p=$ NS), demonstrating that the differential ratio of deletions observed between left and right after hemisection was due mainly to the level of supraspinal inputs. In addition to the modulatory role of supraspinals inputs, we showed that the ratio of deletion diminished statistically with increased treadmill speed after hemisection and spinalization ( $\chi^{2}$ test, $p<0.01$; Fig. $3 B$ ). This result suggests that increasing the level of sensory inputs by augmenting treadmill speed was critical to preventing the failure in St activity.

\section{Discussion}

We show here in SCI cats that, during treadmill locomotion, deletions never occurred when the afferent and supraspinal inputs reaching the spinal locomotor circuitry were normal. In contrast, when the interactions between these systems were perturbed by SCIs, deletions did occur and were dependent on the level of supraspinal and sensory inputs. Such a powerful role of afferent inputs on motoneuronal excitability was shown during treadmill locomotion in premammillary cats (Duysens and Pearson, 1980), which received similar sensory inputs as our cats but supraspinal drive was limited to that originating from brainstem. That study showed that when afferent inputs were normal, deletions never occurred (as in our 
intact cats), whereas when these inputs were perturbed, failures in motoneuronal activity occurred frequently. Accordingly, deletions are more frequently reported during fictive locomotion where sensory inputs are missing (LafreniereRoula and McCrea, 2005; Zhong et al., 2012) and were here reported to decrease with increase treadmill speed, suggesting that afferent inputs play a major role in adjusting the level of motoneuronal excitability through reflex pathways and/or by acting on the CPG. In addition to the role of afferent inputs on spinal excitability, supraspinal inputs also play a strong modulatory role, especially in the context of real locomotion. In our study, we report a higher percentage of deletions on the side of the hemisection compared with the intact side and a higher percentage of deletions after spinalization compared with hemisection, suggesting that the absence of some supraspinal inputs may lead to such deletions. It is indeed known that after a spinal hemisection targeting one side of the cord, asymmetric changes within cutaneous reflex pathways occur within the spinal cord via spared descending pathways (similar dual lesion paradigm as here; Frigon et al., 2009). These results suggest that, in addition to afferent inputs, supraspinal inputs can adjust the motoneuronal excitability by modulating reflex pathways and CPG activity.

Interestingly, the deletions we observed during treadmill locomotion in SCI cats shared some characteristics that were already reported in fictive preparations but also had some interesting specificities. In our study, deletions were always of the nonresetting type and did not perturb the timing of rhythmic activity and bursting characteristics of agonists and antagonists on the ipsilateral and contralateral sides. This supports the hypothesis that each hemicord contains an independent CPG controlling each limb (Zhong et al., 2012) and that each CPG contains a structure that can maintain the periodicity despite specific failures of rhythmic activity in some groups of motoneurons. As suggested by others (Rybak et al., 2006; McCrea and Rybak, 2008), there can be some dissociation between the maintenance of an overall spinal rhythm (rhythm generating network; RG) and the details of the actual output pattern (pattern formation network; PF). According to this model, nonresetting deletions probably result from a spontaneous decrease in excitability of the PF populations projecting to the motoneurons for which activity is "deleted."

In addition, our deletions were only reported in flexor muscles (St or TA), corroborating the hypothesis that the shortest duration phase (swing) is subject to more frequent deletions (McCrea and Rybak, 2008). Moreover, the fact that a single flexor muscle can show a deletion without a concomitant perturbation of the activity in other extensors and flexors has not to our knowledge been reported previously. Indeed, in most "fictive" preparations in which locomotion is evoked by brainstem stimulation (Lafreniere-Roula and McCrea, 2005; Zhong et al., 2012), deletions frequently involve a reduction in the activity of agonists motoneurons throughout the hindlimb, whereas the antagonists exhibit sustained activity in most cases. Such a widespread effect of deletions on the activity of multiple motoneurons throughout the limb strongly suggests that they result from failures of the operation of some common spinal network. This global failure of CPG operation is not seen during real locomotion, probably because the afferent inputs can provide sufficient background excitation of neural elements in the CPG that helps to maintain a certain level of excitability in motoneurons. Despite being less frequent, deletions affecting only one extensor muscle without affecting the other extensors and flexors have been reported during fictive locomotion (Lafreniere-Roula and McCrea, 2005), and such a phenomenon could be reproduced in a computational model of a two-level CPG with an asymmetrical RG in which only the RG flexor population was intrinsically rhythmic (Zhong et al., 2012). This deletion of extensor activity could be produced by a brief removal of excitatory input to the extensor population at the PF level. However, if the same removal of excitatory input was applied to the flexor population, a flexor deletion occurred but the extensors were tonically active. Therefore, although an asymmetric rhythm generator network with a dominant flexor component can explain the finding that nonresetting deletions in extensors do not perturb flexors activity whereas flexor deletions induce rhythm perturbation in extensors (Duysens and Pearson, 1976; Duysens and Pearson, 1980; Zhong et al., 2012), it fails to explain our results showing that a deletion in a flexor muscle can occur without a concomitant failure of extensor muscles. Our results are more consistent with the conclusions reached by McCrea and Rybak (2008), who postulated that the CPG for each limb could have a common RG that may control the operation of multiple unit PF modules (unit burst generator or UBG concept; Grillner, 1981) responsible for the activation of subsets of motoneurons. Such an inclusion of individual PF modules within the two-level CPG would offer more flexibility and is consistent with a broader view of the spinal organization of movement control. There is a large consensus that afferent and descending inputs control many motor behaviors through coordinated activation of specific groups of muscles referred to as synergies (Markin et al., 2012). This suggests that the spinal cord should contain networks of interneurons that activate select motoneuron populations to produce a particular muscle synergy and that these interneurons are targets for descending control and afferent regulation. To summarize, a parallel arrangement of UBGs at the PF layer would allow the failure of one or part of a given UBG without catastrophic consequences on the others and without affecting either the timing at the RG level or the locomotor pattern.

\section{References}

Duysens J, Pearson KG (1976) The role of cutaneous afferents from the distal hindlimb in the regulation of the step cycle of thalamic cats. Exp Brain Res 24:245-255. Medline

Duysens J, Pearson KG (1980) Inhibition of flexor burst generation by loading ankle extensor muscles in walking cats. Brain Res 187:321-332. CrossRef Medline

Frigon A, Barrière G, Leblond H, Rossignol S (2009) Asymmetric changes in cutaneous reflexes after a partial spinal lesion and retention following spinalization during locomotion in the cat. J Neurophysiol 102:26672680. CrossRef Medline

Grillner S (1981) Control of locomotion in bipeds, tetrapods, and fish. In: Handbook of physiology: The nervous system II. (Brookhart JM, Mountcastle VB, eds), pp 1179-1236. Bethesda, MD: American Physiological Society.

Lafreniere-Roula M, McCrea DA (2005) Deletions of rhythmic motoneuron activity during fictive locomotion and scratch provide clues to the organization of the mammalian central pattern generator. J Neurophysiol 94:1120-1132. CrossRef Medline

Markin SN, Lemay MA, Prilutsky BI, Rybak IA (2012) Motoneuronal and muscle synergies involved in cat hindlimb control during fictive and real locomotion: a comparison study. J Neurophysiol 107:2057-2071. CrossRef Medline

Martinez M, Delivet-Mongrain H, Leblond H, Rossignol S (2011) Recovery of hindlimb locomotion after incomplete spinal cord injury in the cat involves spontaneous compensatory changes within the spinal locomotor circuitry. J Neurophysiol 106:1969-1984. CrossRef Medline 
Martinez M, Delivet-Mongrain H, Leblond H, Rossignol S (2012a) Effect of locomotor training in completely spinalized cats previously submitted to a spinal hemisection. J Neurosci 32:10961-10970. CrossRef Medline

Martinez M, Delivet-Mongrain H, Leblond H, Rossignol S (2012b) Incomplete spinal cord injury promotes durable functional changes within the spinal locomotor circuitry. J Neurophysiol 108:124-134. CrossRef Medline

McCrea DA, Rybak IA (2008) Organization of mammalian locomotor rhythm and pattern generation. Brain Res Rev 57:134-146. CrossRef Medline

Pearson KG, Rossignol S (1991) Fictive motor patterns in chronic spinal cats. J Neurophysiol 66:1874-1887. Medline

Rossignol S, Dubuc R, Gossard JP (2006) Dynamic sensorimotor interactions in locomotion. Physiol Rev 86:89-154. CrossRef Medline
Rybak IA, Shevtsova NA, Lafreniere-Roula M, McCrea DA (2006) Modelling spinal circuitry involved in locomotor pattern generation: insights from deletions during fictive locomotion. J Physiol 577:617-639. CrossRef Medline

Stein PS (2008) Motor pattern deletions and modular organization of turtle spinal cord. Brain Res Rev 57:118-124. CrossRef Medline

Stein PS, Daniels-McQueen S (2002) Modular organization of turtle spinal interneurons during normal and deletion fictive rostral scratching. J Neurosci 22:6800-6809. Medline

Zhong G, Shevtsova NA, Rybak IA, Harris-Warrick RM (2012) Neuronal activity in the isolated mouse spinal cord during spontaneous deletions in fictive locomotion: insights into locomotor central pattern generator organization. J Physiol 590:4735-4759. CrossRef Medline 